\title{
More about Neutron Majorana mass from Exotic Instantons: an alternative mechanism in Low-Scale String theory
}

\author{
Andrea Addazi 1 * \\ 1 Dipartimento di Fisica, Università di L'Aquila, 67010 Coppito AQ and LNGS, \\ Laboratori Nazionali del Gran Sasso, 67010 Assergi AQ, Italy
}

\begin{abstract}
We discuss an alternative for Baryon-violating six quarks transitions, in the contest of low scale string theory. In particular, with $M_{S}=10 \div 10^{3} \mathrm{TeV}$, such a transition can be mediated by two colortriplets, through a quartic coupling with down-quarks, generated by exotic instantons, in a calculable and controllable way. We show how FCNCs limits on color-triplet mass are well compatible with $n-\bar{n}$ oscillation ones. If a $n-\bar{n}$ transition were found, this would be an indirect hint for our model. This would strongly motivate searches for direct channels in proton-proton colliders. In fact, our model can be directly tested in a experimentally challenging $100 \div 1000 \mathrm{TeV}$ proton-proton collider, searching for our desired color-triplet states and an evidence for exotic instantons resonances, in addition to stringy Regge resonances, anomalous $Z^{\prime}$-bosons and gauged megaxion. In particular, our scenario can be related to the $750 \mathrm{GeV}$ diphoton hint identifying it with the gauged megaxion dual to the $B$-field. On the other hand, this scenario is compatible with $\mathrm{TeV}$-ish color triplets visible at $\mathrm{LHC}$ and with $1 \div 10 \mathrm{TeV}$ - string scale, i.e stringy resonances at $\mathrm{LHC}$.
\end{abstract}

\section{INTRODUCTION}

The possibility that the string scale can be at much lower energies than the Planck scale $M_{P} \simeq 10^{19} \mathrm{GeV}$, is intriguing and theoretically motivated. In fact, if the string scale was close to the TeV-scale, the hierarchy problem of the Higgs mass would be automatically solved [1 5]. LHC will provide a direct test for TeVscale string theories, searching for stringy Regge resonances as well as massive gluons, massive gravitons, mini black holes. On the other hand, we can argue that also if $M_{S} \simeq 10^{2} \div 10^{3} \mathrm{TeV}$, the hierarchy problem can be much alleviated: $m_{H} / M_{S} \sim 10^{-(3 \div 4)}$ rather than $10^{-14}$. In this case, direct searches at LHC are not possible: stringy resonances would be found in $100 \div 1000 \mathrm{TeV}$ protonproton colliders 17 However, effective operators can be generated, leading to intriguing signatures in low energy physics. Recently, we have shown, how in string-inspired models ${ }^{2}$ non-perturbative effects called exotic stringy instantons can generate new effective operators, violating Baryon and Lepton numbers [8 15. In particular, we have shown how the generation of a Majorana mass term for the neutron from exotic instantons can be possible, without proton destabilization. This leads to the possibility to test indirectly this class of models, in the next generation of experiments on neutron-antineutron oscillations. The actual best limits on $n-\bar{n}$ transition is only $\tau_{n \bar{n}} \simeq 3 \mathrm{yr}$ [21, 23], and the next generation of experiments will enhance this one by two orders of mag-

\footnotetext{
* andrea.addazi@infn.lngs.it

1 In this scenario, also a polynomial running of cross sections with energy and the formation of non-perturbative classical configurations could be detected in future colliders or in UHECR. See [6] for a recent discussion of a string-inspired effective non-local QFT unitarized by classicalization.

${ }^{2}$ See [7] for discussions of other different aspects about stringinspired susy QFT models.
}

nitude [23]. In [8 15], we have shown how proton is not destabilized; but deviations in neutral mesons oscillations and other FCNC are generically predicted, roughly at the same scale as for $n-\bar{n}$ oscillations. As a sequel of our paper, we propose a new alternative mechanism for the generation of a Neutron Majorana mass from exotic instantons, as a variant of the ones discussed in $811^{3}$. In low scale string theory, $M_{S} \simeq 10 \div 10^{3} \mathrm{TeV}$, this mechanism can be tested indirectly in $n-\bar{n}$ experiments and FCNC processes. In particular, we propose a general class of intersecting D-brane models, in which the SM is embedded, and extra color-triplet superfields $C, C^{c}$ naturally emerge for construction. Exotic instantons generate an extra quartic superpotential for $\mathcal{W}_{n p}=C C D^{c} D^{c} / \mathcal{M}_{0}$. As a consequence, a neutron-antineutron transition is induced by non-perturbative effects in a theory of quantum gravity! 4

\section{NEUTRON MAJORANA MASS FROM EXOTIC INSTANTONS}

Let us consider, at effective level, a (N)MSSM plus two extra superfields $C$ and $C^{c} 5 \quad C$ is a $(\overline{3}, 1)_{Y=-2 / 3}$ with respect to $S U(3)_{c} \times S U(2)_{L} \times U(1)_{Y}$, with a Baryon number $B(C)=-2 / 3=2 B\left(D^{c}\right)$. We can introduce at perturbative level the following R-parity preserving

3 In our papers, we have considered a class of exotic instantons wrapping different 3 -cycles with respect to ordinary D-branes. On the other hand, also different classes of exotic instantons, studied in 17-19, may be relevant for phenomenology.

4 For other ideas about neutron-antineutron oscillations in Large Extra Dimensions, see 20.

${ }^{5}$ Even if our model is $\mathcal{N}=1$ supersymmetric, supersymmetry is not necessarly broken at $\mathrm{TeV}$-scale: it can be broken close to the string-scale. 
a)

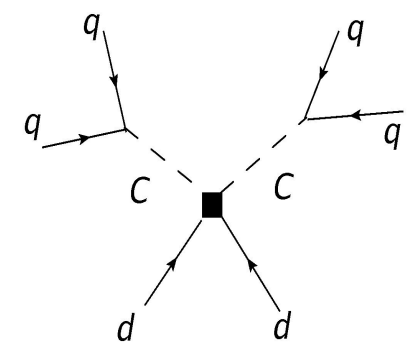

c)

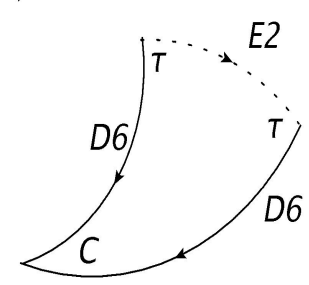

b)

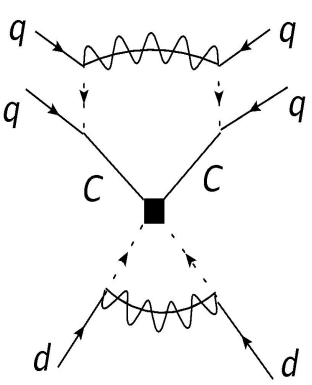

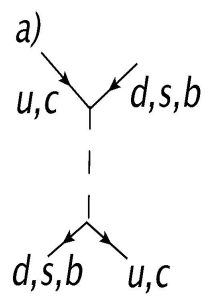
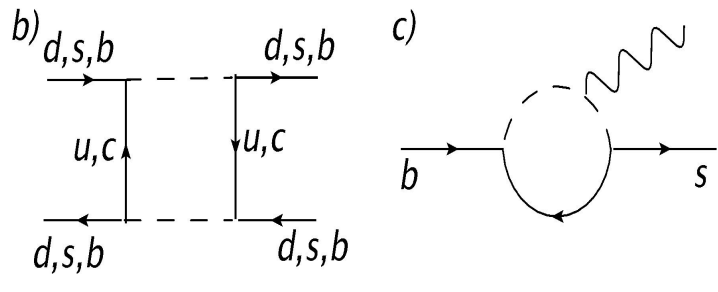

FIG. 2. a) FCNCs tree-level diagrams mediated by $\phi_{C}$ (dashed lines). b) Diagrams of neutral-meson oscillations, mediated by two $\phi_{C}$. c) Diagrams for $b \rightarrow s \gamma$ transition generated by a loop of up-type quarks and a $\phi_{C}$.

the two diagrams dominates, depends on the particular region of the parameters considered. In Fig.1-(c) we show mixed disk amplitudes generating the effective superpotential term (2). We will return later on to the precise calculation of the relevant string amplitudes. Now let us discuss the case in which Fig.1-(a) is dominant with respect Fig.1-(b), i.e supersymmetry is not related to the hierarchy problem of the Higgs mass in this case. Under this assumption, the relevant contributions to FCNCs are shown in Fig.2. In particular, contributions to neutral mesons oscillations as $K^{0}-\bar{K}^{0}$ are generated by box-diagrams. Among all experimental constraints, the strongest one comes from $K^{0}-\bar{K}^{0}$ : this process is suppressed as $\Lambda_{K^{0} \bar{K}^{0}}^{2} \simeq\left(10^{2} \mathrm{TeV}\right)^{2}$ 24]. Assuming $y_{1} \simeq 10^{-2} \div 1$, we can estimate a bound for $m_{\phi_{C}}$ as $m_{\phi_{C}} \simeq 1 \div 1000 \mathrm{TeV}$. On the other hand, a $b \rightarrow s \gamma$ transition is also generated: the experimental suppression approximatively puts the same limits on $m_{\phi_{C}}$. These bounds are higher than the direct ones coming from LHC (roughly near $1 \mathrm{TeV}$ for $y_{1} \sim 1$, as discussed in [9]). We can combine these bounds with the ones from $n-\bar{n}$ oscillation, $\Lambda_{n \bar{n}}>300 \mathrm{TeV}$, and the LHC one on the string scale $M_{S}>1 \div 10 \mathrm{TeV} 25$. The next generation of experiments in $n-\bar{n}$ oscillations promise to test the $1000 \mathrm{TeV}$ scale. This can correspond to (assuming $\left.y_{1} \simeq 1\right) \mathcal{M}_{0} \simeq m_{\phi_{C}} \simeq 10^{3} \mathrm{TeV}$. In this case, FCNC bounds are satisfied. Such a situation can be easily obtained trough exotic instantons. In case in which the diagram in Fig.1-(b) is not sub-dominant, analogous bounds on FCNCs can be obtained, with one more free parameter with than in the one in Fig.1-(a): the gaugino mass. 7.

Let us discuss the mixed disk amplitudes pictures in Fig.1-(c), where string theory enters in our model. $C$ and $Q$ are excitations of open strings stretched between $D 6_{3}-D 6_{3}$ branes and $D 6_{3}-D 6_{2}^{\prime}$-branes respectively. In mixed disk amplitudes, the relevant E2-brane instanton

7 As proposed recently, an auto-concealment of susy can be possible in extra dimensions, with effective Planck scale or stringy scale $1-100 \mathrm{TeV}$ 26]. This scenario generically implies large missing energy channels at LHC. Our proposal can be considered in this contest, in which the supersymmetric diagram is expected to be dominant or at least relevant. 


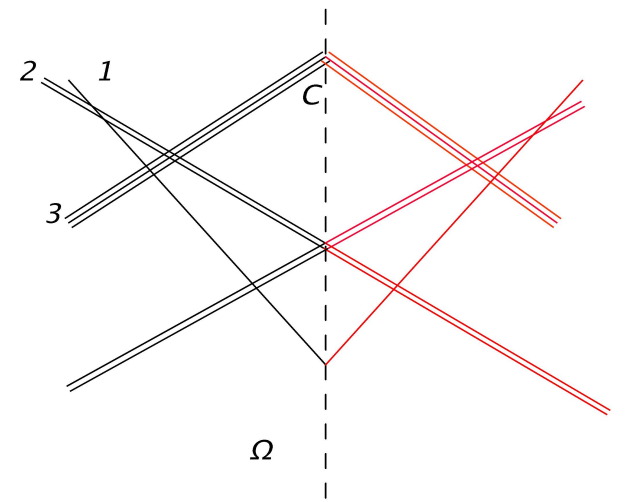

FIG. 3. We show a simplified scheme of a possible class of D-brane systems, generating the fields content of our model. In particular, system shown in figure reproduces $G_{N M S S M}=$ $U(3) \times U(2) \times U(1)$. Clearly, $G_{N M S S M}$ can be extended with one (or more extra) $U(1)$. An antisymmetric $\Omega_{-}$-plane is introduced. $C, C^{c}$ are obtained as excitations of open strings attached to $U(3)_{c}$ and its mirror reflection. Notation: $3 \equiv$ $U(3)_{c}, 2 \equiv U(2), 1 \equiv U(1)$, in black ordinary D-branes, in red "images" of physical D-branes.

intersects two times the $D 6$ and $D 6^{\prime}$ branes. Fermionic modulini $\tau^{i}, \alpha$ are excitations of open string attached to $D 6-E 2$ and $D 6^{\prime}-E 2$ respectively. As a consequence the following effective interactions are generated:

$$
\mathcal{L}_{\text {eff }} \sim D_{i}^{c} \tau^{i} \alpha+C_{i j} \tau^{i} \tau^{j}
$$

We consider a number of intersections of E2-brane and $D 6_{3}-D 6_{2}^{\prime}$ is equal to

$$
I_{E 2-D 6_{3}}=-I_{E 2-D 6_{2}^{\prime}}=2
$$

Integrating over the modulini space (as usually done for istantonic solutions), we obtain

$\mathcal{W}_{n p}=e^{-S_{E 2}} \int d^{6} \tau d^{2} \alpha e^{\mathcal{L}_{e f f}}=\frac{e^{-S_{E 2}}}{M_{S}} \epsilon^{i j k} \epsilon^{l m n} D_{i}^{c} D_{l}^{c} C_{j k} C_{m n}$

So, $\mathcal{M}_{0}=M_{S} e^{+S_{E 2}}$, where $S_{E 2}$ is the effective action of the E2-brane, depending on Kähler moduli associated to 3 -cycles of E2-brane to the Calabi-Yau $C Y_{3}$. Intuitively, if 3-cycles size is small, $e^{S_{E 2}} \sim 1$, on the contrary $e^{S_{E 2}}$ can be much higher than one. For example, a situation in which $\mathcal{M}_{0} \simeq 10^{3} \mathrm{TeV}$ can correspond to $M_{S} \simeq 10^{3} \mathrm{TeV}$ $\left(e^{S_{E 2}} \sim 1\right)$ as well as to $M_{S} \simeq 10 \mathrm{TeV}\left(e^{S_{E 2}} \sim 10^{2}\right)$. We note that exotic instantons have dynamically violated $\mathrm{R}$ parity and the Baryon number, without generating proton or neutralino decays operators.

The effective model proposed can be embedded in several different classes of (NM)SM-like intersecting D-brane systems. See 27 43] for papers and reviews in these subjects, with particular emphasis to possible implications for LHC. A minimal choice can be to consider D-branes systems for $U(3) \times U(2) \times U(1)$. In our construction, we introduce an antisymmetric Mirror $\Omega^{-}$-plane, as shown in Fig.3. This allows to construct $C, C^{c}$ from open strings attached between $U(3)_{c}$ and its mirror twin. In particular, one stack of three D6-branes produces the $U(3)_{c}$ gauge group, including $S U(3)_{c}$ and an extra $U(1)$; one stack of two D6-branes for $U(2)_{L}$, containing $S U(2)_{L}$ and an extra $U(1)$, one stack of a single D6-brane for a $U(1)$ gauge group; an antisymmetric $\Omega$-plane, identifies the D-brane stacks with their images. Let us remind the, $\Omega$-planes explicitly breaks $\mathcal{N}=4$ theory down to $\mathcal{N}=1$ susy theory, and they are usually introduced for tadpole cancellations 45 457 . The presence of orientifold planes $\Omega$ in intersecting D-brane system seems a key element for realistic models of particle physics. Let us note that $U(1)_{3} \subset U(3)_{c}$ and $U(1)_{2} \subset U(2)_{L}$ are anomalous in gauge theories. On the other hand, these $U(1) \mathrm{s}$ are not problematic in string theories. In fact, a generalized Green-Schwarz mechanism can cancel anomalies, through generalized Chern-Simon (GCS) terms. The new vector bosons $Z^{\prime}, Z^{\prime \prime}$ associated to $U(1)_{2,3}$ get masses via Stückelberg mechanisms. See [59 70 for discussions about these aspects in different contests ${ }^{9} 10$. Finally, hypercharge $U(1)_{Y}$ is a non-anomalous linear combination of $U(1)_{3}, U(1)_{2}, U(1)_{1}$ of Fig.3. Our idea is so generic that can be implemented in several different D-brane models. The precise hypercharge combination depends on the particular D-brane construction considered. However, let us note that a complete classification of SM-like D-branes' models are in 89 91. In these models, the presence of extra exotic matter $C, C^{c}$ often seems necessary for a consistent cancellation of all tadpoles and anomalies! So, one can just consider these models in presence of an opportune E2-brane like the one suggested here. Clearly, this un-balances in/out-oriented strings for each stack. However, one can introduce a flavor brane so that the number of in/out oriented strings remains zero for each stack. The construction of a precise quiver theory is not the purpose of this paper, even if we think that just the minimal extension described just above is sufficient. On the other hand, our " $\Omega$-trick" can be considered also in D-brane constructions for models like 3-3-1 as the one considered in 92 94. Usually 3-3-1 models as the one considered in 92 94 are not easily embedded in GUT-inspired models, for its peculiar fields content, while in intersecting D-brane ones there are less difficulties to get such a model, as shown in Ref. $\left[99^{11}{ }^{12}\right.$

\footnotetext{
8 Another important element for intersecting D-brane models can be flavor branes 85 88, but we do not discuss possible explicit model with these ones.

${ }^{9}$ Let us mention that another implementation of the Stückelberg mechanism is in the realization of a Lorentz Violating Massive gravity 71-73]. Recently, geodetic instabilities of Stückelberg Lorentz Violating Massive gravity were discussed in 74]. In subregions of parameters' space of these models, naked singularities are allowed. This could be connected to the existence of new items called frizzyballs in these theories 75 78.

10 Another intriguing application of exotic instantons regards the generation of RH neutrini masses and $\mu$-terms 799 81] 83 84]. This idea is compatible with our one.

${ }^{11}$ I would like to thank José Valle for useful comments on these
} 
Finally, an E2-brane is introduced, intersecting stacks in Fig.3 as indicated in Fig.1-(c), and generating our desiderata superpotential (2).

\section{IMPLICATIONS FOR HIGH ENERGY PROTON-PROTON COLLIDERS}

In this section, we will comment on further implications of our model for high energy colliders.

In particular, the generation of the effective six-quark operator generated in our model has flavor matrix structure. In other words, integrating out $C, C^{c}$ fields for $E<<M_{C}$, our model not only predicts $u d d \rightarrow \bar{u} \bar{d} \bar{d}$ transitions, but also $u d s \rightarrow \bar{u} \bar{d} \bar{s}, \ldots, b u s \rightarrow \bar{b} \bar{u} \bar{s}$ and so on. This implies that for $E_{C M} \simeq M_{C}$, color sextets can decay into two (anti)quarks as

$$
C^{c} \rightarrow u d d, C \rightarrow \bar{u} \bar{d} \bar{d}, \ldots C^{c} \rightarrow b u s, \quad C \rightarrow \bar{b} \bar{u} \bar{s}, \ldots
$$

In general each of these processes are controlled by different couplings, geometrically understood by the mixed disk amplitude structure related to the exotic instanton solution. So that for $M_{C} \simeq 10 \div 100 \mathrm{TeV}$, colored triplets decays can be discovered or limited at LHC or $\sqrt{s}=100-\mathrm{TeV}$ colliders beyond LHC. However, direct research limits have to be compared with indirect measures of FCNC processes as shown before. For instance $y_{1, C u d} C u_{L} d_{L}, y_{1, C c d} C c_{L} d_{L}, y_{1, C u s} C u_{L} s_{L}$ and $y_{1, C c s} C c_{L} s_{L}$ operators are constrained approximately as $y_{1, C u d} y_{1, C u s} \simeq y_{1, C c d} y_{1, C c s} \simeq\left(100 \mathrm{TeV} / \mathrm{m}_{\mathrm{C}}\right)^{2}$. So that, for $m_{C} \simeq 1 \div 10 \mathrm{TeV}$, limits from LHC on $C \rightarrow$ $\bar{u}, \bar{d}, \bar{u} \bar{s}, \bar{c} \bar{d}, \bar{c} \bar{s}$ are less stringent than limits from FCNCs. However, other diagrams involving other generation of quarks are less constrained and processes like $C \rightarrow \bar{t} \bar{b}, \bar{c} \bar{b}$ can be detected by LHC with coupling of the order one. In fact, they can generate $K_{0}-\bar{K}_{0}$ only by extra CKM electroweak loop reductions, i.e., depending on the generation involved, an extra suppression of $10^{-2} \div 10^{-6}$ for the transition amplitude. As regards the exotic instanton, for an effective scale $\Lambda \simeq M_{S} e^{+S_{E 2}}$. A direct test of our model is the detection of the exotic instantons in collisions. As we discussed for $E<<\mathcal{M}_{0} \simeq M_{S} e^{+S_{E 2}}$, the $E 2$-brane is rigidly intersecting the ordinary $D 6$-branes, generating an effective contact interaction. This means that the cross section of the process $q q \rightarrow \bar{q} \bar{q} \bar{q} \bar{q}$ is

$$
\sigma(q q \rightarrow \bar{q} \bar{q} \bar{q} \bar{q})=\left(\operatorname{Tr}\left[y_{1} y_{1}^{\dagger}\right]\right)^{2} \frac{s^{3}}{\mathcal{M}_{0}^{2} m_{C}^{8}}
$$

subjects

${ }^{12}$ Let us also mention that in contest of intersecting D-branes models, an interpretation of dark matter and dark energy as hidden sector is particularly motivated. Recently scenari in which dark matter and dark energy are unified by a hidden strong sector were suggested in Ref. [100 101. In these references, we also commented about possible connections with intersecting D-branes models. for $\sqrt{s}<\left(\mathcal{M}_{0} m_{C}^{4}\right)^{1 / 5}$. For $\sqrt{s}>>\left(\mathcal{M}_{0} m_{C}^{4}\right)^{1 / 5}$, the cross-section (6) is not still valid. For instance, it would violate unitarity. At that scale, the E2-brane cannot more be considered a rigid E-brane and its oscillations are described by fermionic modulini $\tau$ and $\alpha$. At the same scale, the fermionic modulini reggeaize: from the first massless modulini massive Regge states are inevitably excited at this scale, with a Regge slope $\alpha=-1 / 2+\alpha^{\prime} s$. In the region of high energy scattering where all Mandelstam variables are higher than the string scale $\left|s_{i j}\right|>>$ $M_{S}^{2}$ and fixed ratios $s_{i j} / s=$ const, the amplitude will have the universal stringy exponential suppression [103,

$$
\mathcal{A}_{6} \sim e^{-\sum_{i j} \alpha^{\prime} s_{i j} \log \alpha^{\prime} s_{i j}}
$$

Let us note that also at the non-perturbative scale the E2-brane has to conserve its number of intersections which are topological invariants of the mixed disk amplitudes. The production of an exotic instanton in collision is completely different by the production of an elementary particle: while a elementary resonance has a Breit-Wigner amplitude peak, the production of an exotic instanton is expected to be correspond to a highly asymmetric resonance peak: before $\Lambda$ scale the cross section is polynomially increasing while after the cutoff scale $\Lambda$ the cross section is exponentially softened.

Now let us comment on other implications for colliders not directly related to our mechanism for a neutronantineutron transition but inevitably predicted in the class of models considered. In fact, the presence of a supersymmetric scale and a low string scale inevitably leads to the presence of supersymmetric particles and higher spins Regge states. On the other let us note that recent results of LHC disfavor scenari like $M_{S} \simeq 1 \div 10 \mathrm{TeV}$ and $M_{S U S Y} \simeq 1 \div 10 \mathrm{TeV}$. In the contest of our model, the favored region of parameters is $M_{S} \simeq M_{S U S Y} \simeq 100 \mathrm{TeV}$, more naturally compatible with FCNCs and NeutronAntineutron limits. In this scenario, colored supersymmetric partners like gluini, squarks and susy fermionic sextets $\psi_{C}$ are expected to be detectable in $100 \mathrm{TeV}$ proton-proton colliders. On the other hand, massive Regge states like massive gluons, massive gravitons or massive $B_{\mu \nu}$-fields are generically predicted in low scale string theory models. As mentioned in the previous section, the presence of new massive abelian gauge bosons $Z^{\prime}$ interacting through generalized Chern-Simon terms is an avoidable prediction of our class of models. For instance they can decay at tree-level as $Z^{\prime} \rightarrow Z \gamma$, producing a CP-violating polarized photon. Unitarity of $Z^{\prime}$ amplitude imposes the constrain $m_{Z^{\prime}} \simeq M_{S}$. In fact, one-loop correction using vertices $Z^{\prime} Z \gamma$ leads to quadraticaly UV divergent unitarized at the string scale $M_{S}{ }^{13}$. So that, $Z^{\prime}$ can be detected at LHC if and only if $M_{S} \simeq 10 \mathrm{TeV}$.

13 We thank Massimo Bianchi for private communications on these results. 
For a scenario $M_{S} \simeq 100 \mathrm{TeV}, Z^{\prime}$ bosons can be tested in new proton-proton colliders beyond LHC.

Finally, a related implications of the class of model under consideration is the presence of closed strings rank 2 antisymmetric field $B_{\mu \nu}$ dual to a massive axion as $\mathbf{B}=\mathbf{d b}$. In low string scale scenario, the $750 \mathrm{GeV}$ hint measured by ATLAS and CMS can be interpreted as such a gauged axion [102, 105, 107]

\section{CONCLUSIONS AND DISCUSSIONS}

In this paper, we have seen a model for the generation of a Majorana mass from Exotic stringy instantons, as a variant of models proposed in [8, 10? , 11. We have discussed a scenario in which new colortriplet states interact with ordinary quarks, mediating a neutron-antineutron transition. The key of the mechanism is the generation of a non-perturbative quartic interaction among color-triplets and quarks from Exotic Instantons. Proton is not destabilized in this model as well as neutralino or other possible LSP. As a consequence, neutron-antineutron transition can be fast as $\tau_{n \bar{n}} \simeq 300 \mathrm{yr}(1000 \mathrm{TeV})$, in a low scale string theory scenario $M_{S}=10 \div 10^{3} \mathrm{TeV}$. This scenario can be well compatible with FCNC limits, in particular with $K_{0}-\bar{K}_{0}$ and $b \rightarrow s \gamma$. Finally we discussed how this scenario motivates direct researches of colored triplets and exotic instantons in high energy proton-proton colliders. In the Early Universe, new decays $\phi_{C} \rightarrow \bar{q} \bar{q}, q q$, also counting one-loop contributions, could generate a Baryon asymmetry (BAU). However, such a processes could be not enough efficient because of washing-out collisions, generated by exotic instantons, like $d^{c} d^{c} \rightarrow \phi_{C} \phi_{C}$, and by sphalerons successively. On the other hand, string theory suggests that all couplings are dynamical degrees of freedom, stabilized by fluxes and instantons. The scale generated by exotic instantons depends on geometric moduli, associated to the shape of E2-brane cycles wrapping the Calabi-Yau. As a consequence, E2branes Kähler moduli can evolve as dynamical degrees of freedom, so that $e^{-S_{E 2}}($ modulini $)\left[t_{\text {Early-Universe }}\right]<<$

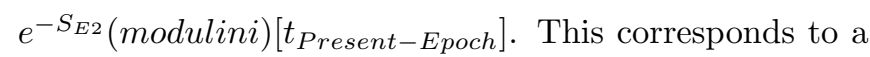
dynamical enlargement of 3 -cycles radii, wrapped in the Calabi-Yau, during the cosmological evolution. For example, geometric moduli can be stabilized to a "solitonic solution" of the Cosmological time $t, \mathcal{F}(t)=$

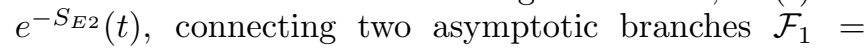
$\mathcal{F}\left(t_{\text {Early-Universe }}\right)$ and $\mathcal{F}_{2}=\mathcal{F}\left(t_{\text {Present-Epoch }}\right)>>\mathcal{F}_{1}$. This is plausible, also considering that usually the dependence on moduli is exponential-like, while a soliton solution is usually a combination of hyperbolic functions, i.e a combination of exponentials. In this way, exotic instantons effects are strongly suppressed during the Early Universe, not washing-out baryon asymmetries, generated after inflation. On the other hand, neutron-antineutron oscillations remain reachable for the next generation of experiments. Such a hypothesis deserves future theoretical and numerical investigations in string phenomenology and baryogenesis calculations.

We conclude that exotic instantons continue to surprise with their intriguing implications in phenomenology. This strongly motivates researches on neutron physics and high energy colliders beyond LHC. This also highly motivates theoretical researches of consistent quivers and Calabi-Yau singularities for the class of D-brane models proposed in our letter, as already done in similar cases 95 98.

\section{Acknowledgments}

I would like to thank the anonymous referee, Massimo Bianchi, Gia Dvali, Cesar Gomez, José Valle and Gabriele Veneziano for useful discussions on these aspects. I also would like to thank Antonino Marcianó and Fudan University for hospitality during the completion of this paper. My work was supported in part by the MIUR research grant Theoretical Astroparticle Physics PRIN 2012CP-PYP7 and by SdC Progetto speciale Multiasse La Societá della Conoscenza in Abruzzo PO FSE Abruzzo 2007-2013.
[1] N. Arkani-Hamed, S. Dimopoulos, G. Dvali (1998). Physics Letters B429 (34): 263272. arXiv:hepph/9803315

[2] I. Antoniadis, N. Arkani-Hamed, S. Dimopoulos, G. Dvali (1998). Physics Letters B436 (34): 257263. arXiv:hep-ph/9804398.

[3] N. Arkani-Hamed, S. Dimopoulos, G. Dvali (1999). Physical Review D59 (8): 086004. arXiv:hep$\mathrm{ph} / 9807344$

[4] L. Randall and R. Sundrum, Phys. Rev. Lett. 83 (1999) 3370 hep-ph/9905221.

[5] L. Randall and R. Sundrum, Nucl. Phys. B 557 (1999) 79 hep-th/9810155.
[6] A. Addazi, arXiv:1505.07357 [hep-th], to appear in the Int.J.Mod.Phys.A.

[7] A. Addazi and G. Esposito, Int. J. Mod. Phys. A 30 (2015) no.15, 1550103 doi:10.1142/S0217751X15501031 arXiv:1502.01471 [hep-th]].

[8] A. Addazi and M. Bianchi, JHEP 1412 (2014) 089 arXiv:1407.2897 [hep-ph]].

[9] A. Addazi, JHEP $1504 \quad$ (2015) 153 doi:10.1007/JHEP04(2015)153 arXiv:1501.04660 [hep-ph]].

[10] A. Addazi and M. Bianchi, JHEP 1507 (2015) 144 arXiv:1502.01531 [hep-ph]].

[11] A. Addazi and M. Bianchi, JHEP 1506 (2015) 012 
arXiv:1502.08041 [hep-ph]]

[12] A. Addazi, arXiv:1505.00625 [hep-ph], to appear in El.J.Th.Physics.

[13] A. Addazi, arXiv:1505.02080 [hep-ph].

[14] A. Addazi, arXiv:1506.06351 [hep-ph], to appear in Phys.Lett.B.

[15] A. Addazi, M. Bianchi and G. Ricciardi, JHEP 1602 (2016) 035 doi:10.1007/JHEP02(2016)035 arXiv:1510.00243 [hep-ph]].

[16] A. Addazi, Nuovo Cim. C 38 (2015) no.1, 21. doi:10.1393/ncc/i2015-15021-6

[17] H. Ghorbani, D. Musso and A. Lerda, JHEP 1103 (2011) 052 arXiv:1012.1122 [hep-th]].

[18] H. Ghorbani and D. Musso, JHEP 1112 (2011) 070 arXiv:1111.0842 [hep-th]].

[19] H. Ghorbani, JHEP 1312 (2013) 041 arXiv:1306.1487 [hep-th]].

[20] S. Nussinov and R. Shrock, Phys. Rev. Lett. 88,171601 (2002).

[21] M. Baldo-Ceolin et al., Z. Phys. C 63 (1994) 409.

[22] K. Abe et al. [Super-Kamiokande Collaboration], Phys. Rev. D 91 (2015) 072006 arXiv:1109.4227 [hep-ex]].

[23] D. G. Phillips, II, W. M. Snow, K. Babu, S. Banerjee, D. V. Baxter, Z. Berezhiani, M. Bergevin and S. Bhattacharya et al., arXiv:1410.1100 [hep-ex]].

[24] Particle Data Group, Phys. Rev. D 86, 010001 (2012).

[25] https://twiki.cern.ch/twiki/bin/view/AtlasPublic/Higgs

[26] S. Dimopoulos, K. Howe, J. March-Russell and J. Scoville, arXiv:1412.0805 [hep-ph].

[27] D. Cremades, L. E. Ibanez and F. Marchesano, JHEP 0307 (2003) 038 hep-th/0302105.

[28] L. A. Anchordoqui, I. Antoniadis, D. C. Dai, W. Z. Feng, H. Goldberg, X. Huang, D. Lust and D. Stojkovic et al., Phys. Rev. D 90 (2014) 6, 066013 arXiv:1407.8120 [hep-ph]].

[29] D. Lust and T. R. Taylor, arXiv:1308.1619 [hep-ph].

[30] L. E. Ibanez, arXiv:1204.5296 [hep-th].

[31] M. Hashi and N. Kitazawa, JHEP 1202 (2012) 050 [JHEP 1204 (2012) 011] arXiv:1110.3976 [hep-ph]].

[32] L. A. Anchordoqui, Adv. High Energy Phys. 2012 (2012) 129879 arXiv:1108.5694 [hep-ph]].

[33] M. Berasaluce-Gonzalez, L. E. Ibanez, P. Soler and A. M. Uranga, JHEP 1112 (2011) 113 arXiv:1106.4169 [hep-th]].

[34] M. Cvetic, J. Halverson and R. Richter, arXiv:0910.2239 [hep-th].

[35] M. Cvetic, J. Halverson and R. Richter, JHEP 1007 (2010) 005 arXiv:0909.4292 [hep-th]].

[36] L. A. Anchordoqui, H. Goldberg, D. Lust, S. Stieberger and T. R. Taylor, Mod. Phys. Lett. A 24 (2009) 2481 arXiv:0909.2216 [hep-ph]].

[37] J. A. Maxin, V. E. Mayes and D. V. Nanopoulos, Phys. Rev. D 81 (2010) 015008 arXiv:0908.0915 [hep-ph]].

[38] J. A. Maxin, V. E. Mayes and D. V. Nanopoulos, Phys. Rev. D 81 (2010) 015008 arXiv:0908.0915 [hep-ph]].

[39] V. Rosenhaus and W. Taylor, JHEP 0906 (2009) 073 arXiv:0905.1951 [hep-th]].

[40] L. E. Ibanez and R. Richter, JHEP 0903 (2009) 090 arXiv:0811.1583 [hep-th]].

[41] J. Kumar, Phys. Rev. D $\mathbf{7 9} \quad$ (2009) 066007 arXiv:0808.1264 [hep-th]].

[42] D. Lust, S. Stieberger and T. R. Taylor, Nucl. Phys. B 808 (2009) 1 arXiv:0807.3333 [hep-th]].

[43] D. Berenstein, R. Martinez, F. Ochoa and S. Pinansky,
Phys. Rev. D 79 (2009) 095005 arXiv:0807.1126 [hep$\mathrm{ph}]$.

[44] A. Sagnotti, IN *CARGESE 1987, PROCEEDINGS, NONPERTURBATIVE QUANTUM FIELD THE-

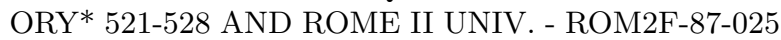
(87,REC.MAR.88) 12p hep-th/0208020.

[45] A. Sagnotti, Phys. Lett. B294 (1992) 196 hepth/9210127.

[46] C. Angelantonj and A. Sagnotti, Phys. Rept. 1 [(Erratum-ibid.) 339] arXiv:hep-th/0204089

[47] G. Pradisi and A. Sagnotti, Phys. Lett. B 216 (1989) 59.

[48] M. Bianchi and A. Sagnotti, Phys. Lett. B247 (1990) 517

[49] M. Bianchi, G. Pradisi and A. Sagnotti, Nucl. Phys. B376 (1992) 365.

[50] D. Fioravanti, G. Pradisi and A. Sagnotti, Phys. Lett. B321 (1994) 349 arXiv:hep-th/9311183.

[51] A. Sagnotti, in *Palaiseau 1995, Susy 95* 473-484 hepth/9509080

[52] A. Sagnotti, Nucl. Phys. Proc. Suppl. 56B (1997) 332 arXiv:hep-th/9702093.

[53] A. M. Uranga, Nucl. Phys. B 598, 225 (2001) hepth/0011048.

[54] G. Aldazabal, S. Franco, L. E. Ibanez, R. Rabadan and A. M. Uranga, J. Math. Phys. 42, 3103 (2001) hepolicReshyttono11073.

[55] M. Bianchi and J. F. Morales, JHEP 0003 (2000) 030 hep-th/0002149.

[56] C. Angelantonj, M. Bianchi, G. Pradisi, A. Sagnotti and Y. .S. Stanev, Phys. Lett. B 385 (1996) 96 hepth/9606169.

[57] C. Angelantonj, M. Bianchi, G. Pradisi, A. Sagnotti and Y. S. Stanev, Phys. Lett. B 387 (1996) 743 hepth/9607229.

[58] M. Bianchi, S. Cremonesi, A. Hanany, J. F. Morales, D. R. Pacifici and R. K. Seong, JHEP 1410 (2014) 27 arXiv:1408.1957 [hep-th]].

[59] P. Anastasopoulos, M. Bianchi, E. Dudas and E. Kiritsis, JHEP 0611 (2006) 057 hep-th/0605225.

[60] J. De Rydt, J. Rosseel, T.T. Schmidt, A. Van Proeyen and M. Zagermann, Class. Quant. Grav. 24 (2007) 5201 arXiv:0705.4216] [INSPIRE].

[61] D. Feldman, Z. Liu and P. Nath, Phys. Rev. D 75 (2007) 115001 hep-ph/0702123 [INSPIRE].

[62] D. Feldman, Z. Liu and P. Nath, AIP Conf. Proc. 939 (2007) 50 arXiv:0705.2924 [INSPIRE].

[63] B. Körs and P. Nath, Phys. Lett. B 586 (2004) 366 hep-ph/0402047. [INSPIRE].

[64] B. Körs and P. Nath, JHEP 12 (2004) 005 hepph/0406167] [INSPIRE].

[65] B. Körs and P. Nath, hep-ph/0411406 [INSPIRE].

[66] B. Körs and P. Nath, JHEP 07 (2005) 069 hepph/0503208] [INSPIRE].

[67] P. Anastasopoulos, F. Fucito, A. Lionetto, G. Pradisi, A. Racioppi and Y.S. Stanev, Phys. Rev. D 78 (2008) 085014 arXiv:0804.1156. [INSPIRE].

[68] C. Corianó, N. Irges and E. Kiritsis, Nucl. Phys. B 746 (2006) 77 hep-ph/0510332 [INSPIRE].

[69] M. Bianchi and E. Kiritsis, Nucl. Phys. B 782 (2007) 26 hep-th/0702015.

[70] B. de Wit, P.G. Lauwers and A. Van Proeyen, Nucl. Phys. B 255 (1985) 569 [INSPIRE].

[71] N. Arkani-Hamed, H. Georgi and M. D. Schwartz, An- 
nals Phys. 305 (2003) 96.

[72] V. A. Rubakov, arXiv:hep-th/0407104

[73] S. L. Dubovsky, JHEP 0410, 076 (2004).

[74] A. Addazi and S. Capozziello, Int. J. Theor. Phys. 54 (2015) 6, 1818 arXiv:1407.4840 [gr-qc]].

[75] A. Addazi, arXiv:1508.04054 [gr-qc].

[76] A. Addazi, arXiv:1510.05876 [gr-qc], to appear in the proceeding of KSM 15, Frankfurt.

[77] A. Addazi, Electron. J. Theor. Phys. 12 (2015) no.34, 89.

[78] A. Addazi, arXiv:1603.08719 [gr-qc], to appear in Int.J.Geom.Meth.Phys.

[79] R. Blumenhagen, M. Cvetic and T. Weigand, Nucl. Phys. B 771 (2007) 113 hep-th/0609191.

[80] L.E. Ibanez , A.M. Uranga, JHEP 0703 (2007) 052.

[81] L.E. Ibanez , A.N. Schellekens, A.M. Uranga, JHEP 0706 (2007) 011.

[82] S. Antusch, L.E. Ibanez and T. Macr, JHEP 0709 (2007) 087.

[83] R. Blumenhagen, M. Cvetic, D. Lust, R. Richter and T. Weigand, Phys. Rev. Lett. 100 (2008) 061602 arXiv:0707.1871 [hep-th]].

[84] H. Abe, T. Kobayashi, Y. Tatsuta and S. Uemura, arXiv:1502.03582 [hep-ph].

[85] G. Aldazabal, D. Badagnani, L. E. Ibanez, and A. Uranga, JHEP 9906 (1999) 031, arXiv:hep-th/9904071 [hep-th].

[86] M. Bianchi and J. F. Morales, JHEP 03 (2000) 030, hep-th/0002149.

[87] A.M. Uranga, Nucl.Phys. B598 (2001) 225-246, arXiv:hep-th/0011048 [hep-th].

[88] M.Bianchi, G.Inverso, J. F. Morales, D. R. Pacifici JHEP 1401 (2014) 128, arXiv:1307.0466.

[89] M. Cvetic, J. Halverson and R. Richter, arXiv:0910.2239 [hep-th].

[90] M. Cvetic, J. Halverson and P. Langacker, JHEP 1111 (2011) 058 arXiv:1108.5187 [hep-ph]].

[91] J. Halverson, Phys. Rev. Lett. 111 (2013) 26, 261601
arXiv:1310.1091 [hep-th]].

[92] S. M. Boucenna, S. Morisi and J. W. F. Valle, Phys. Rev. D 90 (2014) 1, 013005 arXiv:1405.2332 [hep-ph]].

[93] S. M. Boucenna, J. W. F. Valle and A. Vicente, arXiv:1502.07546 [hep-ph].

[94] S. M. Boucenna, R. M. Fonseca, F. Gonzalez-Canales and J. W. F. Valle, Phys. Rev. D 91 (2015) 3, 031702 arXiv:1411.0566 [hep-ph]].

[95] G. Aldazabal, L. E. Ibanez, F. Quevedo, and A. M. Uranga, JHEP 08 (2000) 002, arXiv:hep-th/0005067

[96] D. Berenstein, V. Jejjala, and R. G. Leigh, Phys.Rev.Lett. 88 (2002) 071602, arXiv:hepph/0105042 [hep-ph].

[97] G. Aldazabal, L. E. Ibanez, and F. Quevedo, JHEP 0002 (2000) 015, arXiv:hep-ph/0001083 [hep-ph].

[98] J. Cascales, M. Garcia del Moral, F. Quevedo, and A. Uranga, JHEP 0402 (2004) 031, arXiv:hep-th/0312051 [hep-th].

[99] A. Addazi, J. W. F. Valle and C. A. Vaquera-Araujo, arXiv:1604.02117 [hep-ph].

[100] A. Addazi, P. Don and A. Marcian, arXiv:1602.01772 [gr-qc].

[101] A. Addazi, A. Marciano and S. Alexander, arXiv:1603.01853 [gr-qc].

[102] L. E. Ibanez and V. Martin-Lozano, arXiv:1512.08777 [hep-ph].

[103] D. J. Gross and P. F. Mende, Nucl. Phys. B 303 (1988) 407.

[104] L. A. Anchordoqui, I. Antoniadis, H. Goldberg, X. Huang, D. Lust and T. R. Taylor, arXiv:1603.08294 [hep-ph].

[105] T. Li, J. A. Maxin, V. E. Mayes and D. V. Nanopoulos, arXiv:1602.09099 [hep-ph].

[106] P. Anastasopoulos and M. Bianchi, arXiv:1601.07584 [hep-th].

[107] L. A. Anchordoqui, I. Antoniadis, H. Goldberg, X. Huang, D. Lust and T. R. Taylor, arXiv:1603.08294 [hep-ph]. 